\title{
Birational types of algebraic orbifolds
}

Kresch, Andrew ; Tschinkel, Yuri

\begin{abstract}
We introduce a variant of the birational symbols group of Kontsevich, Pestun and the second author, and use this to define birational invariants of algebraic orbifolds.
\end{abstract}

DOI: https://doi.org/10.1070/sm9386

Posted at the Zurich Open Repository and Archive, University of Zurich ZORA URL: https://doi.org/10.5167/uzh-205949

Journal Article

Accepted Version

Originally published at:

Kresch, Andrew; Tschinkel, Yuri (2021). Birational types of algebraic orbifolds. Sbornik: Mathematics, 212(3):319-331.

DOI: https://doi.org/10.1070/sm9386 


\title{
BIRATIONAL TYPES OF ALGEBRAIC ORBIFOLDS
}

\author{
ANDREW KRESCH AND YURI TSCHINKEL
}

\begin{abstract}
We introduce a variant of the birational symbols group of Kontsevich, Pestun, and the second author, and use this to define birational invariants of algebraic orbifolds.
\end{abstract}

\section{INTRODUCTION}

Let $k$ be a field of characteristic zero and $X$ a smooth projective variety over $k$, of dimension $n$; we require our varieties to be irreducible, but not necessarily geometrically irreducible. The paper [15] introduced the Burnside group of varieties

$$
\operatorname{Burn}_{n}=\operatorname{Burn}_{n, k},
$$

the free abelian group on isomorphism classes of finitely generated fields of transcendence degree $n$ over $k$; for such a field $K$ we denote the corresponding generator by $[K]$. To $X$ one associates its class

$$
[X]:=[k(X)] \in \operatorname{Burn}_{n},
$$

extended by additivity for general smooth projective schemes. To

$$
U \subset X \backslash D,
$$

the complement to a simple normal crossing divisor

$$
D=D_{1} \cup \cdots \cup D_{\ell},
$$

one may also associate a class in $\operatorname{Burn}_{n}$ :

$$
[U]:=[X]-\sum_{1 \leq i \leq \ell}\left[D_{i} \times \mathbb{P}^{1}\right]+\sum_{1 \leq i<j \leq \ell}\left[\left(D_{i} \cap D_{j}\right) \times \mathbb{P}^{2}\right]-\ldots
$$

This is not only an invariant of the isomorphism type of $U$, but is a birational invariant in the following sense: $[U]=\left[U^{\prime}\right]$ in $\operatorname{Burn}_{n}$ if there exist a quasiprojective variety $V$ and birational projective morphisms

$$
V \rightarrow U \quad \text { and } \quad V \rightarrow U^{\prime} .
$$

This formalism was used to establish specialization of rationality.

Date: November 4, 2019. 
Now we suppose that $X$ is equipped with a faithful action of a finite abelian group $G$. Then Burn $_{n}$ is replaced by the equivariant Burnside group

$$
\operatorname{Burn}_{n}(G) \text {, }
$$

introduced in [14] (in a slightly different form, as explained in Appendix A). This is the quotient by suitable relations of the free abelian group on triples consisting of:

- a subgroup $H \subset G$,

- a $G / H$-Galois algebra extension $K$ of a field $K_{0}$ of transcendence degree $d \leq n$ over $k$, up to equivariant isomorphism, required to satisfy Assumption 1 in Appendix A (a technical condition, always satisfied when $k$ is algebraically closed), and

- a faithful $(n-d)$-dimensional linear representation of $H$ over $K_{0}$ with trivial space of invariants, up to equivalence.

Then an invariant of the $G$-equivariant birational type of $X$ is obtained from a stratification of $X$ by the stabilizer group $H \subset G$ as the sum

$$
\sum_{H \subset G} \sum_{\substack{Y \subset X \\ \text { with stabilizer } H}}\left[\left(G / H \subset k(Y), \beta_{Y}(X)\right],\right.
$$

where the inner sum is over unions $Y$ of $G$-orbits of components, with $k(Y)$ understood as the product of function fields of the components of $Y$, and where $\beta_{Y}(X)$ records the generic normal bundle representation along $Y$. There is an analogous $G$-equivariant birational invariant of $U$ as above, where each $D_{i}$ is assumed to be $G$-invariant.

This paper concerns birational invariants of (quasi)projective orbifolds $\mathcal{X}$. Here, by an orbifold we mean a smooth separated irreducible finitetype Deligne-Mumford stack over $k$ with trivial generic stabilizer. Such a stack has a coarse moduli space [12], a separated algebraic space of finite type over $k$. Following [16], we say that the orbifold $\mathcal{X}$ is quasiprojective when the coarse moduli space is a quasiprojective variety, and is projective when the coarse moduli space is a projective variety. By Theorems 4.4 and 5.3 of op. cit., every quasiprojective orbifold may be presented as a locally closed substack of a projective orbifold.

We will introduce a variant

$$
\overline{\operatorname{Burn}}_{n}
$$

of the groups $\operatorname{Burn}_{n}$ and $\operatorname{Burn}_{n}(G)$. In essence, we only carry in $\overline{\operatorname{Burn}}_{n}$ the information of representations of finite abelian groups, up to automorphisms of those groups. Working with $\overline{\operatorname{Burn}}_{n}$, we exhibit a birational invariant of a quasiprojective $n$-dimensional orbifold $\mathcal{X}$. 
It suffices to consider finite abelian groups thanks to the divisorialification procedure of [6], a sequence of blow-ups in smooth centers which, when applied to a general orbifold, yields an orbifold with only abelian groups as geometric stabilizer groups. Weak factorization [2], in a functorial form proved in [3], is used to exhibit the desired birational invariance.

In Section 2 we recall the Burnside group of varieties and establish a presentation by scissors-like relations, analogous to the scissors relations defining the Grothendieck group of varieties. Section 3 introduces the orbifold version $\overline{\mathrm{Burn}}_{n}$, where the birational invariant of quasiprojective orbifolds defined in Section 4 takes its value (Theorem 4.1). A computation of invariants of orbifold surfaces reveals an intriguing connection with modular curves (Proposition 4.3), whose proof is given in Section 5.

Acknowledgments: The first author was partially supported by the Swiss National Science Foundation.

\section{Burnside Group VIA SCISSORS RELATIONS}

Let $k$ be a field of characteristic zero. The Grothendieck group

$$
\mathrm{K}_{0}\left(\operatorname{Var}_{k}\right)
$$

may be approached in two ways, as an abelian group generated by the classes of algebraic varieties over $k$ with the classical scissors relations (where it makes no difference if we restrict to just smooth quasiprojective varieties), or via the Bittner presentation [8], which only involves smooth projective varieties. Of course, $\mathrm{K}_{0}\left(\operatorname{Var}_{k}\right)$ has a ring structure as well, but we do not concern ourselves with this here.

In this section we record the observation that the Burnside group $\operatorname{Burn}_{n}$ also admits a description in terms of scissors relations. As mentioned in the Introduction, we only require our varieties to be irreducible (but not necessarily geometrically irreducible).

Lemma 2.1. Let $k$ be a field of characteristic zero, and let $W$ be a smooth quasiprojective variety over $k$. For any nonempty open $U \subset W$ there exist divisors $D_{1}, \ldots, D_{\ell}$ such that $W \backslash D_{1}$ is contained in $U$, and $D_{1} \backslash D_{2}, \ldots, D_{\ell-1} \backslash D_{\ell}, D_{\ell}$ are all smooth.

Proof. Let $Z=W \backslash U$. By [13, Thm. 7], given an embedding of $W$ in projective space, a general hypersurface of sufficiently large degree containing $Z$ defines a divisor $D_{1}$ on $W$ whose singular locus $D_{1}^{\text {sing }}$ is contained in $Z$ and does not contain any irreducible component of $Z$. If $D_{1}$ is smooth, then we are done with $\ell=1$. Otherwise, we have $\operatorname{dim}\left(D_{1}^{\text {sing }}\right)<\operatorname{dim}(Z)$, and we conclude by induction on $\operatorname{dim}(Z)$. 
Proposition 2.2. Let $k$ be a field of characteristic zero and $n$ a natural number. Then the assignment to $[k(X)]$ of $[X]$ for smooth projective varieties $X$ of dimension $n$ over $k$ defines an isomorphism

$$
\operatorname{Burn}_{n} \stackrel{\sim}{\longrightarrow}\left(\bigoplus_{[U], \operatorname{dim}(U)=n} \mathbb{Z} \cdot[U]\right) / \text { modified-scissors, }
$$

where, on the right, we have the quotient of the free abelian group on isomorphism classes of smooth quasiprojective varieties of dimension $n$ over $k$ by the modified scissors relations

$$
[U]=\left[V \times \mathbb{P}^{n-d}\right]+[U \backslash V]
$$

for smooth closed subvarieties $V \subset U$ of dimension $d<n$. The inverse isomorphism is given by the formula (1.1).

Proof. We check that the map from the statement of the proposition is well-defined, i.e., the classes of any pair of birationally equivalent smooth projective $n$-dimensional varieties are equal modulo the modified scissors relations.

By weak factorization, it suffices to consider the case of $X$ and $B \ell_{Y} X$, where $X$ is smooth and projective of dimension $n$ and $Y$ is a smooth subvariety of $X$ of dimension $d<n$. We have

$$
\begin{aligned}
{[X] } & =\left[Y \times \mathbb{P}^{n-d}\right]+[X \backslash Y], \\
{\left[B \ell_{Y} X\right] } & =\left[\mathbb{P}\left(N_{Y / X}\right) \times \mathbb{P}^{1}\right]+[X \backslash Y],
\end{aligned}
$$

where $N_{Y / X}$ denotes the normal bundle. We are done if we can show that $\left[\mathbb{P}\left(N_{Y / X}\right) \times \mathbb{P}^{1}\right]=\left[Y \times \mathbb{P}^{n-d}\right]$. We will show, more generally, that for any smooth quasiprojective variety $W$ of dimension $e<n$ and vector bundle $F$ on $W$ of rank $r \leq n+1-e$, we have

$$
\left[\mathbb{P}(F) \times \mathbb{P}^{n+1-e-r}\right]=\left[W \times \mathbb{P}^{n-e}\right] .
$$

For any smooth quasiprojective variety $Z$ of dimension $n-1$ we have $\left[Z \times \mathbb{A}^{1}\right]=0$ (by considering $Z \times\{\infty\} \subset Z \times \mathbb{P}^{1}$ ), and hence

$$
\left[W \times \mathbb{P}^{n-e}\right]=\left[W \times\left(\mathbb{P}^{1}\right)^{n-e}\right]
$$

(by considering $W \times \mathbb{P}^{n-e-1} \subset W \times \mathbb{P}^{n-e}$ ). We prove (2.1) by induction on $e$; the case $e=0$ is now clear. Let $U \subset W$ be a nonempty open subset on which $F$ is trivial, and $D_{1}, \ldots, D_{\ell}$, divisors as in Lemma 2.1. The modified scissors relation and the induction hypothesis lead to

$$
\begin{aligned}
{\left[\mathbb{P}(F) \times \mathbb{P}^{n+1-e-r}\right]=[} & \left.D_{\ell} \times \mathbb{P}^{n+1-e}\right]+\left[\left(D_{\ell-1} \backslash D_{\ell}\right) \times \mathbb{P}^{n+1-e}\right] \\
+\cdots+ & {\left[\left(D_{1} \backslash\left(D_{2} \cup \cdots \cup D_{\ell}\right) \times \mathbb{P}^{n+1-e}\right]\right.} \\
& +\left[\left(W \backslash\left(D_{1} \cup \cdots \cup D_{\ell}\right)\right) \times \mathbb{P}^{n-e}\right]
\end{aligned}
$$


We conclude with the relations, for $1 \leq i \leq \ell$ :

$$
\begin{aligned}
{\left[\left(W \backslash\left(D_{i+1} \cup \cdots \cup D_{\ell}\right)\right) \times \mathbb{P}^{n-e}\right]=} & {\left[\left(D_{i} \backslash\left(D_{i+1} \cup \cdots \cup D_{\ell}\right)\right) \times \mathbb{P}^{n+1-e}\right] } \\
& +\left[\left(W \backslash\left(D_{i} \cup \cdots \cup D_{\ell}\right)\right) \times \mathbb{P}^{n-e}\right] .
\end{aligned}
$$

Now we verify that the map in the reverse direction, given by the formula (1.1), is well-defined, i.e., respects the modified scissors relations. Let $V$ be a smooth closed subvariety of $U$ of dimension $d$. Then $U$ may be presented as the complement in a smooth projective variety $X$ of a simple normal crossing divisor $D_{1} \cup \cdots \cup D_{\ell}$, with which a smooth subvariety $Y \subset X$ has normal crossing, such that $Y \cap U=V$. We have $[U]$, given

by the formula (1.1). For $\left[V \times \mathbb{P}^{n-d}\right]$ we have the embedding in $Y \times \mathbb{P}^{n-d}$, complement to the simple normal crossing divisor

$$
\left(D_{1} \cap Y\right) \times \mathbb{P}^{n-d} \cup \cdots \cup\left(D_{\ell} \cap Y\right) \times \mathbb{P}^{n-d},
$$

and thus an analogous formula in $\operatorname{Burn}_{n}$. The blow-up $B \ell_{Y} X$ has the simple normal crossing divisor $\widetilde{D}_{1} \cup \cdots \cup \widetilde{D}_{\ell} \cup E$, where $\widetilde{D}_{i}$ denotes the proper transform of $D_{i}$, and $E$, the exceptional divisor, leading to a formula for $[U \backslash V]$ in Burn $_{n}$. Comparing formulas and using that any intersection not involving $E$ is birational to the corresponding intersection in $X$, while any intersection involving $E$ is birational to the product of an intersection in $Y$ with projective space of the appropriate dimension, we obtain the desired relation.

That the composite of the forward and reverse maps, in either order, is the identity, is clear for the composite $\operatorname{Burn}_{n} \rightarrow \operatorname{Burn}_{n}$, and for the other, comes down to iterated application of the modified scissors relations.

\section{BuRNSIDE GROUP FOR STACKS}

In this section we introduce a variant of the equivariant Burnside group which is adapted to the setting of orbifolds.

Definition 3.1. We define the $\mathbb{Z}[t]$-module $\overline{\mathcal{B}}$ by starting with the free $\mathbb{Z}$-module on pairs $(A, S)$ consisting of a finite abelian group $A$ and finite generating system $S$ of $A$, where the action of $t$ is to append the element 0 to $S$, and passing to the quotient by the following relations:

- $(A, S)$ and $\left(A, S^{\prime}\right)$ are equivalent if $S^{\prime}$ is a permutation of $S$.

- $(A, S)$ and $\left(A^{\prime}, S^{\prime}\right)$ are equivalent if some isomorphism $A \cong A^{\prime}$ transforms $S$ to $S^{\prime}$. 
- $(A, S), S=\left(a_{1}, \ldots, a_{m}\right)$, is equivalent, for any $2 \leq j \leq m$, to

$$
\begin{aligned}
& \sum_{\emptyset \neq I \subset\{1, \ldots, j\}}(-t)^{|I|-1}\left(A /\left\langle a_{i}-a_{i_{0}}\right\rangle_{i \in I},\right. \\
& \left.\left.\quad\left(\bar{a}_{i_{0}}, \bar{a}_{1}-\bar{a}_{i_{0}}, \ldots \text { (omitting all } i \in I\right) \ldots, \bar{a}_{j}-\bar{a}_{i_{0}}, \bar{a}_{j+1}, \ldots, \bar{a}_{m}\right)\right),
\end{aligned}
$$

where inside the sum $i_{0}$ denotes an element of $I$, with sequence of elements of $A /\left\langle a_{i}-a_{i_{0}}\right\rangle_{i \in I}$ of length $1+(j-|I|)+(m-j)$ that is independent of the choice of $i_{0}$.

We let $[A, S]$ denote the class in $\overline{\mathcal{B}}$ of a pair $(A, S)$. The natural grading on $\mathbb{Z}[t]$ yields a grading on $\overline{\mathcal{B}}$ that assigns degree $|S|$ to $[A, S]$ :

$$
\overline{\mathcal{B}}=\bigoplus_{n=0}^{\infty} \overline{\mathcal{B}}_{n}
$$

Representations determine elements of $\overline{\mathcal{B}}$ : if $G$ is a finite diagonalizable group scheme with representation

$$
\rho: G \rightarrow G L_{n}
$$

(over an arbitrary field), then there is an associated element

$$
[\rho] \in \overline{\mathcal{B}}_{n},
$$

given by the Cartier dual group, with the sequence of weights supplied by a decomposition of $\rho$ as a sum of $n$ one-dimensional linear representations.

Restricting to $e$-torsion groups $A$ for a positive integer $e$, respectively, to $p$-primary $A$ for a prime number $p$, leads to a $\mathbb{Z}[t]$-module $\overline{\mathcal{B}}^{[e]}$, respectively $\overline{\mathcal{B}}^{(p)}$. The evident homomorphisms from these modules to $\overline{\mathcal{B}}$ are split monomorphisms, with splittings given by

$$
[A, S] \rightarrow[A / e A, S], \quad \text { respectively, } \quad[A, S] \rightarrow[A(p), S],
$$

where $A(p)$ denotes the $p$-primary subgroup of $A$. We have

$$
\overline{\mathcal{B}}=\bigoplus_{p} \overline{\mathcal{B}}^{(p)}, \quad \overline{\mathcal{B}}^{(p)}=\underset{j}{\lim } \overline{\mathcal{B}}^{\left[p^{j}\right]}
$$

Definition 3.2. Let $k$ be a field of characteristic zero and $n$ a natural number. The group

$$
\overline{\operatorname{Burn}}_{n}
$$

is the abelian group generated by pairs $(K, \alpha)$, where

- $K$ is a field of transcendence degree $d \leq n$ over $k$ and

- $\alpha \in \overline{\mathcal{B}}_{n-d}$,

modulo the identification of $(K(t), \beta)$ and $(K, t \beta)$ for $\beta \in \overline{\mathcal{B}}_{n-d-1}$. 
Example 3.3. For $\overline{\mathcal{B}}_{2}^{[5]}$ we have generators $t^{2}[0,()], t\left[C_{5},(1)\right],\left[C_{5},(1,1)\right]$, $\left[C_{5},(1,2)\right],\left[C_{5},(1,4)\right],\left[C_{5} \oplus C_{5},((1,0),(0,1))\right]$, and relations:

$$
\begin{aligned}
t\left[C_{5},(1)\right] & =\left[C_{5},(1,4)\right]+t\left[C_{5},(1)\right]-t^{2}[0,()], \\
{\left[C_{5},(1,1)\right] } & =2 t\left[C_{5},(1)\right]-t\left[C_{5},(1)\right], \\
{\left[C_{5},(1,2)\right] } & =\left[C_{5},(1,1)\right]+\left[C_{5},(1,2)\right]-t^{2}[0,()], \\
{\left[C_{5},(1,4)\right] } & =2\left[C_{5},(1,2)\right]-t^{2}[0,()], \\
{\left[C_{5} \oplus C_{5},((1,0),(0,1))\right] } & =2\left[C_{5} \oplus C_{5},((1,0),(0,1))\right]-t\left[C_{5},(1)\right],
\end{aligned}
$$

where $C_{5}=\mathbb{Z} / 5 \mathbb{Z}$. We deduce

$\left[C_{5} \oplus C_{5},((1,0),(0,1))\right]=\left[C_{5},(1,1)\right]=\left[C_{5},(1,4)\right]=t\left[C_{5},(1)\right]=t^{2}[0,()]$,

with

$$
2\left(\left[C_{5},(1,2)\right]-t^{2}[0,()]\right)=0 .
$$

As may be seen directly, or by application of Theorem 4.1, below, over an algebraically closed field of characteristic zero, among rational orbifold surfaces whose only nontrivial stabilizer groups have order 5 , the parity of the number of isolated points with $C_{5}$-stabilizer and unequal weights not summing to zero remains invariant under blow-ups of points. As noted in [5, Exa. 4.3], it is not possible to eliminate such an isolated point with $C_{5}$-stabilizer just with blow-ups of points.

\section{Birational inVARIANTS OF ORBIFOLDS}

In this section we introduce new birational invariants of $n$-dimensional orbifolds over a field $k$ of characteristic zero, taking values in $\overline{\operatorname{Burn}}_{n}$.

Let $\mathcal{X}$ be an orbifold. We recall from [5] (see also [6]): if $D_{1} \cup \cdots \cup D_{\ell}$ is a simple normal crossing divisor on $\mathcal{X}$, then $\mathcal{X}$ is called divisorial with respect to $D_{1}, \ldots, D_{\ell}$ if the morphism

$$
\mathcal{X} \rightarrow B \mathbb{G}_{m}^{\ell}
$$

determined by $\mathcal{O}_{\mathcal{X}}\left(D_{i}\right)$, for $i=1, \ldots, \ell$, is representable. We will apply this terminology more generally to any a finite collection of line bundles.

Divisorialification is a procedure that, when applied to an orbifold $\mathcal{X}$, yields a succession of blow-ups along smooth centers

$$
\mathcal{Y} \rightarrow \cdots \rightarrow \mathcal{X}
$$

such that $\mathcal{Y}$ is divisorial with respect to a suitable simple normal crossing divisor. This is given as Algorithm $\mathrm{C}$ in [5], initially with a requirement to have abelian geometric stabilizer groups, later with this requirement removed [6].

As explained in the introduction, invariance under birational projecive morphisms is the statement of invariance under the equivalence relation 
of existence of a third object (variety or Deligne-Mumford stack) with birational projective morphisms to two given objects. In this section we are interested in quasiprojective orbifolds $\mathcal{X}$ and $\mathcal{X}^{\prime}$, and the equivalence takes the form of existence of a Deligne-Mumford stack $\mathcal{Y}$ with birational projective morphisms

$$
\mathcal{Y} \rightarrow \mathcal{X} \quad \text { and } \quad \mathcal{Y} \rightarrow \mathcal{X}^{\prime}
$$

There is no loss of generality in supposing $\mathcal{Y}$ as well to be an orbifold, since resolution of singularities in a functorial form as in [22] and [7] is applicable to algebraic stacks. Here we remind the reader that when $\mathcal{X}$ and $\mathcal{Y}$ are quasiprojective orbifolds, a representable morphism $\mathcal{Y} \rightarrow \mathcal{X}$ is projective if and only if it is proper. (Every projective morphism is proper. The reverse implication uses that $\mathcal{Y} \rightarrow \mathcal{X}$ factors up to a 2isomorphism through $\mathcal{X} \times_{X} Y$, where $X$ and $Y$ denote the respective coarse moduli spaces, that $\mathcal{X} \rightarrow X$ and $\mathcal{Y} \rightarrow Y$ induce bijections on geometric points, and that a representable proper morphism inducing a bijection on geometric points is finite, hence projective.)

Theorem 4.1. Let $k$ be a field of characteristic zero, $n$ a natural number, and $\mathcal{X}$ an $n$-dimensional quasiprojective orbifold over $k$. The following recipe, assigning to $\mathcal{X}$ a class $[\mathcal{X}] \in \overline{\operatorname{Burn}}_{n}$ gives an invariant under representable birational projective morphisms:

- Use divisorialification to replace $\mathcal{X}$ by a quasiprojective orbifold $\mathcal{Y}$ that is divisorial with respect to some finite collection of line bundles.

- Stratify $\mathcal{Y}$ by the isomorphism type of the geometric stabilizer group and attach to each component the normal bundle:

$$
\mathcal{Y}=\coprod_{G} \mathcal{Y}_{G}, \quad N_{Y, G}=N_{\mathcal{Y}_{G} / \mathcal{Y}}
$$

- Writing the coarse moduli space of $\mathcal{Y}_{G}$, for each $G$, as $Y_{G}$, we assign the element

$$
[\mathcal{X}]:=\sum_{G}\left(\left[Y_{G}\right],\left[N_{Y, G}\right]\right) \in \overline{\operatorname{Burn}}_{n} .
$$

In the last step, if $Y_{G}$ is irreducible of dimension d, then we understand $\left[Y_{G}\right]$ to be the associated element of $\operatorname{Burn}_{d}$, with $\left[N_{Y, G}\right] \in \overline{\mathcal{B}}_{n-d}$ associated to the representation of $G$ at the geometric generic point of $\mathcal{Y}_{G}$. In general, we understand $\left(\left[Y_{G}\right],\left[N_{Y, G}\right]\right)$ to be the sum of the elements of $\overline{\mathrm{Burn}}_{n}$ attached to the irreducible components. 
Proof. Let $\mathcal{X}^{\prime}$ be a quasiprojective orbifold with representable birational projective morphism to $\mathcal{X}$. We divisorialize $\mathcal{X}^{\prime}$ to obtain $\mathcal{Y}^{\prime}$. The diagram

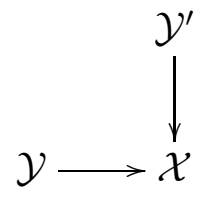

may be completed to a 2-commutative square of representable birational projective morphisms of quasiprojective orbifolds by desingularizing the closure in the fiber product of a nonempty open substack where the morphisms are isomorphisms. This way, we are reduced to showing that for a representable birational projective morphism $\mathcal{Z} \rightarrow \mathcal{Y}$ of quasiprojective orbifolds we have

$$
\sum_{G}\left(\left[Y_{G}\right],\left[N_{Y, G}\right]\right)=\sum_{G}\left(\left[Z_{G}\right],\left[N_{Z, G}\right]\right) \in \overline{\operatorname{Burn}}_{n} .
$$

Let $\mathcal{L}_{1}, \ldots, \mathcal{L}_{\ell}$ be line bundles, relative to which $\mathcal{Y}$ is divisorial. The functorial form of weak factorization in [3] is applicable to stacks and yields a factorization of $\mathcal{Z} \rightarrow \mathcal{Y}$ as a composite of maps of divisorial projective orbifolds (with respect to pullbacks of $\mathcal{L}_{1}, \ldots, \mathcal{L}_{\ell}$ ), each equal to or inverse to a blow-up along a smooth center.

Let $\mathcal{V}$ be a smooth closed substack of $\mathcal{Y}$ of dimension $d<n$, with coarse moduli space $V$, and let $\mathcal{Z}=B \ell_{\mathcal{V}} \mathcal{Y}$. We verify (4.1) in this case. On the left, we break up $\mathcal{Y}_{G}$ into the unions of components $\mathcal{Y}_{G}^{\prime}$ disjoint from $\mathcal{V}$ and $\mathcal{Y}^{\prime \prime}$, meeting $\mathcal{V}$ nontrivially, and apply the modified scissors relation to $\mathcal{Y}_{G}^{\prime \prime}$ :

$$
\begin{aligned}
& \sum_{G}\left(\left[Y_{G}\right],\left[N_{Y, G}\right]\right)=\sum_{G}\left(\left[Y_{G}^{\prime}\right],\left[N_{Y, G}\right]\right) \\
& +\sum_{G}\left(\left[Y_{G}^{\prime \prime} \cap V, t^{\operatorname{dim}\left(\mathcal{Y}_{G}^{\prime \prime}\right)-\operatorname{dim}\left(\mathcal{Y}_{G}^{\prime \prime} \cap \mathcal{V}\right)}\left[N_{Y, G}\right]\right)+\sum_{G}\left(\left[Y_{G}^{\prime \prime} \backslash V\right],\left[N_{Y, G}\right]\right),\right.
\end{aligned}
$$

where in the second sum on the right, the dimensions are understood to be taken componentwise. Breaking up the sum on the right of (4.1) in a similar fashion, we obtain an expression with identical first and third sums and a second sum that differs from the second sum in the expression above by relations in $\overline{\mathcal{B}}$.

Example 4.2. Functorial destackification [5] of an orbifold provides a sequence of blow-ups along smooth centers and root stack operations along smooth divisors that simplify the stack structure. The root stack operation adds stabilizer $\mu_{n}$ (for some positive integer $n$ ) along a divisor $[10, \S 2],[1$, App. B], and the outcome of destackification is an orbifold that is obtained from a smooth variety by iterating root stack operations 


\begin{tabular}{rcrcrc}
$p$ & $\overline{\mathcal{B}}_{2}^{[p]} /\left(\mathcal{C} \cap \overline{\mathcal{B}}_{2}^{[p]}\right)$ & $p$ & $\overline{\mathcal{B}}_{2}^{[p]} /\left(\mathcal{C} \cap \overline{\mathcal{B}}_{2}^{[p]}\right)$ & $p$ & $\overline{\mathcal{B}}_{2}^{[p]} /\left(\mathcal{C} \cap \overline{\mathcal{B}}_{2}^{[p]}\right)$ \\
\hline 5 & $\mathbb{Z} / 2 \mathbb{Z}$ & 17 & $\mathbb{Z} / 2 \mathbb{Z} \oplus \mathbb{Z}$ & 31 & $\mathbb{Z}^{2}$ \\
7 & 0 & 19 & $\mathbb{Z}$ & 37 & $\mathbb{Z} / 2 \mathbb{Z} \oplus \mathbb{Z}^{2}$ \\
11 & $\mathbb{Z}$ & 23 & $\mathbb{Z}^{2}$ & 41 & $\mathbb{Z} / 2 \mathbb{Z} \oplus \mathbb{Z}^{3}$ \\
13 & $\mathbb{Z} / 2 \mathbb{Z}$ & 29 & $\mathbb{Z} / 2 \mathbb{Z} \oplus \mathbb{Z}^{2}$ & 43 & $\mathbb{Z}^{3}$
\end{tabular}

TABLE 1. Isomorphism type of $\overline{\mathcal{B}}_{2}^{[p]} /\left(\mathcal{C} \cap \overline{\mathcal{B}}_{2}^{[p]}\right)$

along components of a simple normal crossing divisor. Blow-ups alone are, as noted in Example 3.3, insufficient to bring a general orbifold into this form. Correspondingly, we view the quotient $\overline{\mathcal{B}} / \mathcal{C}$, where $\mathcal{C}$ denotes the submodule generated by the classes of pairs

$$
\left(C_{a_{1}} \oplus \cdots \oplus C_{a_{r}},\left(g_{1}, \ldots, g_{r}\right)\right)
$$

of direct sums of finite cyclic groups and tuples of generators, as an invariant of an orbifold up to smooth blow-ups and root stacks. We have

$$
\overline{\mathcal{B}}^{[p]} \subset \mathcal{C} \quad \text { for } \quad p \in\{2,3\},
$$

since blow-ups suffice for the destackification in these cases [17], [20].

Table 1 , which records the isomorphism type of $\overline{\mathcal{B}}_{2}^{[p]} /\left(\mathcal{C} \cap \overline{\mathcal{B}}_{2}^{[p]}\right)$ for some primes $p \geq 5$, reveals a pattern that we are able to confirm.

Proposition 4.3. For a prime $p \geq 5$ let

$$
g=g\left(X_{0}(p)\right)
$$

denote the genus of the modular curve, i.e.,

$$
g= \begin{cases}{\left[\frac{p}{12}\right] \mp 1,} & \text { when } p \equiv \pm 1 \bmod 12 \\ {\left[\frac{p}{12}\right],} & \text { otherwise }\end{cases}
$$

Then

$$
\overline{\mathcal{B}}_{2}^{[p]} /\left(\mathcal{C} \cap \overline{\mathcal{B}}_{2}^{[p]}\right) \cong \begin{cases}\mathbb{Z} / 2 \mathbb{Z} \oplus \mathbb{Z}^{g}, & \text { if } p \equiv 1 \bmod 4 \\ \mathbb{Z}^{g}, & \text { if } p \equiv 3 \bmod 4 .\end{cases}
$$

The proof of Proposition 4.3, based on computations with Manin's modular symbols [18], is given in the next section.

The entry 0 in Table 1 for $p=7$ indicates that $\overline{\mathcal{B}}_{2}^{[7]} \subset \mathcal{C}$. In fact, we have $\overline{\mathcal{B}}_{3}^{[7]} \subset \mathcal{C}$ as well. But we find

$$
\overline{\mathcal{B}}_{4}^{[7]} /\left(\mathcal{C} \cap \overline{\mathcal{B}}_{4}^{[7]}\right) \cong \mathbb{Z} / 2 \mathbb{Z} .
$$




\section{Modular Symbols And the Proof of Proposition 4.3}

The equivariant Burnside group introduced in [14] is shown to exhibit a tantalizing connection with the modular curves $X_{1}(N)$ for various $N$. Here, we see the appearance of the modular curves

$$
X_{0}(p)=\Gamma_{0}(p) \backslash \mathbb{H} \cup\{0, \infty\}
$$

and the corresponding modular symbols [18].

Fix a prime $p \geq 5$; we are interested in the isomorphism type of the abelian group

$$
\overline{\mathcal{B}}_{2}^{[p]} /\left(\mathcal{C} \cap \overline{\mathcal{B}}_{2}^{[p]}\right)
$$

with generators

$$
\left[C_{p},(1, a)\right], \quad 2 \leq a \leq p-2,
$$

and relations

$$
\begin{aligned}
{\left[C_{p},(1, a)\right] } & =\left[C_{p},\left(1, a^{-1}\right)\right] \quad \text { for all } a, \\
2\left[C_{p},(1,2)\right] & =0, \\
{\left[C_{p},(1,2)\right] } & =-\left[C_{p},(1, p-2)\right], \\
{\left[C_{p},(1, a)\right] } & =\left[C_{p},(1, a-1)\right]+\left[C_{p},\left(1, a^{-1}-1\right)\right] \\
& \quad \text { for } a \in\left\{3, \ldots, \frac{p-1}{2}\right\} \cup\left\{\frac{p+3}{2}, \ldots, p-2\right\},
\end{aligned}
$$

where $a^{-1}$ denotes the positive integer less than $p$, inverse to $a \bmod p$.

The modular group

$$
\Gamma_{0}(p)=\left\{\left(\begin{array}{ll}
a & b \\
c & d
\end{array}\right) \in \mathrm{SL}_{2}(\mathbb{Z}) \mid c \equiv 0 \bmod p\right\}
$$

has index $p+1$ in $\mathrm{SL}_{2}(\mathbb{Z})$, with right coset representatives

$$
\left(\begin{array}{ll}
1 & 0 \\
0 & 1
\end{array}\right),\left(\begin{array}{ll}
1 & 0 \\
1 & 1
\end{array}\right), \ldots,\left(\begin{array}{cc}
1 & 0 \\
p-1 & 1
\end{array}\right),\left(\begin{array}{cc}
0 & -1 \\
1 & 0
\end{array}\right) .
$$

We let $\Gamma_{0}(p)$ act in the standard way on the upper half-plane $\mathbb{H}$ and as well on $\mathbb{Q} \cup\{i \infty\}$, the latter with two orbits corresponding to the cusps $0, \infty \in X_{0}(p)$. Here, 0 corresponds to the set of all $b / d \in \mathbb{Q}$ with $p \nmid d$ and $\infty$, to the set of $a / c \in \mathbb{Q}$ with $p \mid c$. The real structure on $X_{0}(p)$ is determined by the standard complex conjugation $\mathbb{H} \rightarrow \mathbb{H}, z \mapsto-\bar{z}$. It is well known that the real locus of $X_{0}(p)$ is connected.

With Manin's modular symbols [18], applied to $\Gamma_{0}(p)$, we get a presentation of $H_{1}\left(X_{0}(p), \mathbb{Z}\right)$ by generators and relations. Proposition 4.3 is established by showing that these relations, together with the additional relations that the sum of any cycle and its complex conjugate is zero, match the presentation (5.1)-(5.4). In fact, we use a simpler set of relations, which yield the homology not of the Riemann surface $X_{0}(p)$, but 
rather of the corresponding orbifold curve which carries orbifold structure at elliptic points. The quotient of $\mathbb{H}$ by $\Gamma_{0}(p) /\{ \pm 1\}$ is an orbifold, compactified by adding the cusps to obtain the orbifold curve

$$
X_{0}(p)_{\text {orb }}
$$

Orbifolds and their topological invariants are explained, for instance, in [19], while a convenient reference for orbifold curves is [4]. However, $H_{1}\left(X_{0}(p)_{\text {orb }}, \mathbb{Z}\right)$ may also be presented directly as the homology of the complement of the elliptic points, modulo the relation that an appropriate multiple of a small loop around an elliptic point is zero. When $p \equiv 1 \bmod 4$ there is a complex conjugate pair of elliptic points of $H_{1}\left(X_{0}(p)_{\text {orb }}, \mathbb{Z}\right)$ where the stabilizer (of a representative point of $\mathbb{H}$ ) has order 2 in $\Gamma_{0}(p) /\{ \pm 1\}$; for each of these, twice a small loop is declared to be zero in homology. When $p \equiv 1 \bmod 6$ there is a complex conjugate pair of elliptic points where the stabilizer has order 3 in $\Gamma_{0}(p) /\{ \pm 1\}$, for which we declare 3 times a small loop to be zero in homology.

We summarize the needed results from [18], modified appropriately to the orbifold setting. We maintain the convention from (5.1)-(5.4) about $a$ and $a^{-1}$ and, when $a \notin\{p-2,(p-1) / 2\}$ define positive integers $a^{\prime}$ and $a^{\prime \prime}$ less than $p$ by the requirements

$$
a^{\prime} \equiv-a^{-1}-1 \bmod p, \quad a^{\prime \prime} \equiv-(a+1)^{-1} \bmod p .
$$

Lemma 5.1 ([18, (1.4)]). A surjective homomorphipsm

$$
\Gamma_{0}(p) \rightarrow H_{1}\left(X_{0}(p)_{\text {orb }}, \mathbb{Z}\right)
$$

is defined by sending $\gamma \in \Gamma_{0}(p)$ to the image

$$
\{0, \gamma \cdot 0\}
$$

in $X_{0}(p)$ of a geodesic path in $\mathbb{H} \cup \mathbb{Q}$ from 0 to $\gamma \cdot 0$. The kernel is generated by the commutator subgroup of $\Gamma_{0}(p)$ and the parabolic elements of $\Gamma_{0}(p)$.

Lemma $5.2([18,(1.5)-(1.9)])$. The abelian group $H_{1}\left(X_{0}(p)_{\text {orb }}, \mathbb{Z}\right)$ is presented by generators

$$
\left\{0, \frac{1}{a}\right\}, \quad 2 \leq a \leq p-2,
$$

and relations

$$
\begin{aligned}
\left\{0, \frac{1}{a}\right\}+\left\{0, \frac{1}{p-a^{-1}}\right\} & =0, \\
\left\{0, \frac{1}{a}\right\}+\left\{0, \frac{1}{a^{\prime}}\right\}+\left\{0, \frac{1}{a^{\prime \prime}}\right\} & =0, \\
\left\{0, \frac{1}{(p-1) / 2}\right\}+\left\{0, \frac{1}{p-2}\right\} & =0 .
\end{aligned}
$$


Now the proof of Proposition 4.3 combines an algebraic result with topological reasoning.

Lemma 5.3. An isomorphism

$$
\begin{aligned}
\overline{\mathcal{B}}_{2}^{[p]} /\left(\mathcal{C} \cap \overline{\mathcal{B}}_{2}^{[p]}\right) \rightarrow & \\
& \left.H_{1}\left(X_{0}(p)_{\text {orb }}, \mathbb{Z}\right) /\left\langle\left\{0, \frac{1}{a}\right\}+\left\{0, \frac{1}{p-a}\right\}, a \in\{2, \ldots, p-2\}\right\}\right\rangle
\end{aligned}
$$

is given by $\left[C_{p},(1, a)\right] \mapsto\{0,1 / a\}$ for all $a$.

Proof. Suppose $2 \leq b \leq(p-3) / 2$. We subtract the relations (5.4) corresponding to $a=b+1$ and $a=p-b$, noticing that the rightmost terms cancel thanks to (5.1), to obtain

$$
\left[C_{p},(1, b+1)\right]-\left[C_{p},(1, p-b)\right]=\left[C_{p},(1, b)\right]-\left[C_{p},(1, p-b-1)\right] .
$$

Starting from (5.3) we obtain, inductively,

$$
\left[C_{p},(1, a)\right]=-\left[C_{p},(1, p-a)\right]
$$

for all $a$. Using (5.8) and (5.1), we rewrite (5.4) as

$$
\left[C_{p},(1, a)\right]+\left[C_{p},\left(1, a^{\prime}\right)\right]+\left[C_{p},\left(1, a^{\prime \prime}\right)\right]=0
$$

for $a \notin\{(p-1) / 2, p-2\}$. We conclude by matching relations (5.1)(5.2), (5.8)-(5.9) with (5.5)-(5.7) and the additional relations from the quotient group in the statement of the lemma.

While $H_{1}\left(X_{0}(p), \mathbb{Z}\right)$ is free of rank $2 g$ (where $g$ is the genus of $X_{0}(p)$ ), there may be torsion in $H_{1}\left(X_{0}(p)_{\text {orb }}, \mathbb{Z}\right)$ :

$$
H_{1}\left(X_{0}(p)_{\text {orb }}, \mathbb{Z}\right) \cong \begin{cases}\mathbb{Z} / 6 \mathbb{Z} \oplus \mathbb{Z}^{2 g}, & \text { if } p \equiv 1 \bmod 12, \\ \mathbb{Z} / 2 \mathbb{Z} \oplus \mathbb{Z}^{2 g}, & \text { if } p \equiv 5 \bmod 12, \\ \mathbb{Z} / 3 \mathbb{Z} \oplus \mathbb{Z}^{2 g}, & \text { if } p \equiv 7 \bmod 12, \\ \mathbb{Z}^{2 g}, & \text { if } p \equiv 11 \bmod 12 .\end{cases}
$$

Complex conjugation acts on $H_{1}\left(X_{0}(p)_{\text {orb }}, \mathbb{Z}\right)$ by

$$
\left\{0, \frac{1}{a}\right\} \mapsto\left\{0, \frac{1}{p-a}\right\} \text {. }
$$

Lemma 5.3 identifies $\overline{\mathcal{B}}_{2}^{[p]} /\left(\mathcal{C} \cap \overline{\mathcal{B}}_{2}^{[p]}\right)$ with the quotient of $H_{1}\left(X_{0}(p)_{\text {orb }}, \mathbb{Z}\right)$ by the elements of the form sum of a cycle and its conjugate.

Complex conjugation acts trivially on $H_{1}\left(X_{0}(p)_{\text {orb }}, \mathbb{Z}\right)_{\text {tors }}$. When $p \equiv 1$ mod 4 , intersection number mod 2 with a conjugation-invariant curve joining the order 2 elliptic points splits off $H_{1}\left(X_{0}(p)_{\text {orb }}, \mathbb{Z}\right)[2]$ equivariantly as a direct summand of $H_{1}\left(X_{0}(p)_{\text {orb }}, \mathbb{Z}\right)$. Now $\overline{\mathcal{B}}_{2}^{[p]} /\left(\mathcal{C} \cap \overline{\mathcal{B}}_{2}^{[p]}\right)$ is a direct sum of $\mathbb{Z} / 2 \mathbb{Z}$ when $p \equiv 1 \bmod 4$, zero when $p \equiv 3 \bmod 4$, and the 
quotient of $H_{1}\left(X_{0}(p), \mathbb{Z}\right)$ by the elements of the form sum of a cycle and its conjugate. The latter is accessed by choosing a conjugation-invariant triangulation of $X_{0}(p)$ and using spectral sequences relating the equivariant homology of $X_{0}(p)$ with the group homology of $H_{j}\left(X_{0}(p), \mathbb{Z}\right)$, on the one hand, and the group homology of the groups of $j$-chains on the other, for $j=0,1,2$; cf. [9, $\S$ VII.7]. (All group homology is for the group $\mathbb{Z} / 2 \mathbb{Z}$, corresponding to complex conjugation.) We omit the details and report only the outcome:

$$
\begin{aligned}
H_{i}\left(\mathbb{Z} / 2 \mathbb{Z}, H_{1}\left(X_{0}(p), \mathbb{Z}\right)\right)=0 \quad \text { for all } i \geq 1, \\
H_{j}^{\mathbb{Z} / 2 \mathbb{Z}}\left(X_{0}(p), \mathbb{Z}\right) \cong \begin{cases}\mathbb{Z}, & \text { if } j=0, \\
\mathbb{Z} / 2 \mathbb{Z} \oplus \mathbb{Z}^{g}, & \text { if } j=1, \\
\mathbb{Z} / 2 \mathbb{Z}, & \text { if } j \geq 2 .\end{cases}
\end{aligned}
$$

The vanishing of $H_{1}\left(\mathbb{Z} / 2 \mathbb{Z}, H_{1}\left(X_{0}(p), \mathbb{Z}\right)\right)$ has the consequence that the subgroup of $H_{1}\left(X_{0}(p), \mathbb{Z}\right)$ of elements of the form sum of a cycle and its conjugate is saturated, i.e., has torsion-free quotient.

\section{Appendix A. G-EQuivariant Burnside Group}

Let $G$ be a finite abelian group, with character group $A$, and $X$ a smooth projective variety over an algebraically closed field of characteristic zero, with a faithful action of $G$. The paper [14] introduced

- the abelian group

$$
\mathcal{B}_{n}(G)
$$

as the quotient of the $\mathbb{Z}$-module generated by symbols $\left(a_{1}, \ldots, a_{n}\right)$, with $a_{i} \in A$, and subject to conditions and relations similar to those in Definition 3.1,

- the equivariant Burnside group

$$
\operatorname{Burn}_{n}(G)
$$

and

- the $G$-equivariant birational invariant

$$
\beta(X) \in \operatorname{Burn}_{n}(G) \text {. }
$$

The invariant $\beta(X)$ is the term corresponding to $H=G$ in the first sum in the formula (1.2) from the Introduction, with some indices shifted by 1. The shift of indices reflects that, under the assumption that $G$ is nontrivial and the fixed locus $X^{G}$ is nonempty, $X^{G}$ has positive codimension in $X$. In this Appendix we explain in detail the formula (1.2).

Now, let $k$ be an arbitrary field of characteristic zero and $X$ a smooth projective variety over $k$, with a faithful action of a finite abelian group $G$. Let $H \subset G$ be a subgroup. The $H$-fixed locus $X^{H}$ is smooth, a finite 
union of orbits of components. After removing those where the generic point has stabilizer strictly larger than $H$ we are left with $Y_{1}, \ldots, Y_{r}$, each with an induced faithful action of the quotient group $G / H$. Let $Z_{1}, \ldots, Z_{r}$ denote the respective quotient varieties. With the convention from the Introduction, by which we may write $k(Y)$ when $Y$ is not necessarily irreducible, we have a $G / H$-Galois algebra extension $k\left(Y_{i}\right) / k\left(Z_{i}\right)$ for every $i$.

Assumption 1. For all $H$ and all $k\left(Y_{i}\right) / k\left(Z_{i}\right)$, the field $k\left(Z_{i}\right)$ contains primitive $e$ th roots of unity, where $e$ is the exponent of $H$, and the homomorphism

$$
H^{1}\left(G, k\left(Y_{i}\right)^{\times}\right) \rightarrow H^{1}\left(H, k\left(Y_{i}\right)^{\times}\right)^{G / H}=H^{1}\left(H, k\left(Z_{i}\right)^{\times}\right)
$$

of the Hochschild-Serre spectral sequence is surjective.

The homomorphism in Assumption 1 is always injective, since by the Hochschild-Serre spectral sequence the kernel is $H^{1}\left(G / H, k\left(Y_{i}\right)^{\times}\right)$, which vanishes by Hilbert's Theorem 90 (for Galois algebras, this may be found in $[11, \S 4.3])$. Thus, Assumption 1 implies that it is an isomorphism.

The next result tells us that, that under Assumption 1, for every $n$ the non-abelian cohomology set $H^{1}\left(G, G L_{n}\left(k\left(Y_{i}\right)\right)\right.$ ) (see, e.g., [21, §1.3.2]) may be identified with $H^{1}\left(H, G L_{n}\left(k\left(Z_{i}\right)\right)\right)$, the set of equivalence classes of linear $n$-dimensional representations of $H$ over $k\left(Z_{i}\right)$.

Proposition A.1. Let $G$ be a finite abelian group, $H \subset G$ a subgroup, $K_{0}$ a field containing a primitive eth root of unity, where e denotes the exponent of $H$, and $K a G / H$-Galois algebra over $K_{0}$. If the homomorphism $H^{1}\left(G, K^{\times}\right) \rightarrow H^{1}\left(H, K_{0}^{\times}\right)$of the Hochschild-Serre spectral sequence is surjective, then for every positive integer $n$ there is a unique bijective map of non-abelian cohomology sets

$$
H^{1}\left(G, G L_{n}(K)\right) \rightarrow H^{1}\left(H, G L_{n}\left(K_{0}\right)\right)
$$

that is compatible with restriction

$$
H^{1}\left(G, G L_{n}(K)\right) \rightarrow H^{1}\left(H, G L_{n}(K)\right)
$$

and extension of scalars

$$
H^{1}\left(H, G L_{n}\left(K_{0}\right)\right) \rightarrow H^{1}\left(H, G L_{n}(K)\right) .
$$

Proof. We fix a primitive eth root of unity $\zeta \in K_{0}$. The extension of scalars map from the statement is injective (by standard representation theory), so it suffices to exhibit a compatible bijective map of the indicated non-abelian cohomology sets. In fact, it suffices to verify the compatibility condition after replacing $H^{1}\left(H, G L_{n}(K)\right)$ with $H^{1}\left(H, G L_{n}(\widehat{K})\right)$ for some étale extension $\widehat{K} / K$. 
By the structure theorem of finite abelian groups,

$$
H \cong \mathbb{Z} / n_{1} \mathbb{Z} \times \cdots \times \mathbb{Z} / n_{r} \mathbb{Z},
$$

for $n_{1}, \ldots, n_{r} \geq 2$ with $n_{i} \mid n_{i+1}$ for $i=1, \ldots, r-1$; we have $e=n_{r}$. Let $\zeta \in K_{0}$ be a primitive $e$ th root of unity. Sending the $i$ th generator of $H$ to $\zeta^{e / n_{i}}$ and all other generators to 1 , we have an element of $H^{1}\left(H, K_{0}^{\times}\right)$ which, by hypothesis, comes from a 1-cocycle $\left(u_{i, g}\right)_{g \in G}$ with values in $K^{\times}$. As remarked above, the homomorphism from the statement is always injective, therefore $\left(u_{i, g}^{n_{i}}\right)_{g \in G}$ is a 1-coboundary, i.e., for some $v_{i} \in K^{\times}$we have

$$
u_{i, g}^{n_{i}}={ }^{g} v_{i} / v_{i} \quad \text { for all } \quad g \in G .
$$

The data of $\left(u_{i, g}^{n_{i}}\right)_{i, g}$ and $\left(v_{i}\right)_{i}$ give us a way to assign, functorially, a $H$ Galois algebra over an étale $K_{0}$-algebra $L_{0}$ to every $G$-Galois $L / L_{0}$ with $G$-equivariant $K_{0}$-algebra homomorphism $K \rightarrow L$. Specifically, given $G$ Galois $L / L_{0}$ with $\iota: K \rightarrow L$ we apply Hilbert's Theorem 90 to obtain $w_{i} \in L^{\times}$for every $i$, satisfying $\iota\left(u_{i, g}\right)={ }^{g} w_{i} / w_{i}$ for all $g$. Now $\iota\left(v_{i}\right) w_{i}^{-n_{i}}$ is Galois-invariant, i.e., lies in $L_{0}$, and is unique up to multiplication by an element of $\left(L_{0}^{\times}\right)^{n_{i}}$, for every $i$ (since $w_{i}$ is unique up to multiplication by an element of $\left.L_{0}\right)$; we associate the $H$-Galois algebra

$$
L_{0}\left[t_{1}, \ldots, t_{r}\right] /\left(t_{1}^{n_{1}}-\iota\left(v_{1}\right) w_{1}^{-n_{1}}, \ldots, t_{r}^{n_{r}}-\iota\left(v_{r}\right) w_{r}^{-n_{r}}\right) .
$$

The functorial association is fully faithful. We deduce that it is essentially surjective, hence gives an equivalence of categories, using that any $H$ Galois $L_{0}^{\prime} / L_{0}$ is trivialized by an étale extension of $L_{0}$ (e.g., $L_{0}^{\prime} \otimes_{L_{0}} L_{0}^{\prime} \cong$ $\prod_{h \in H} L_{0}^{\prime}$ ) and described up to isomorphism by an $H$-valued 1-cocycle (in Galois cohomology).

Finally, the non-abelian cohomology sets from the statement are invariants of the categories described in the previous paragraph. A unique element of $H^{1}\left(G, G L_{n}(K)\right)$, respectively, $H^{1}\left(H, G L_{n}\left(K_{0}\right)\right)$, is associated to a functorial association of a free $L_{0}$-module of rank $n$ to every $G$-Galois $L / L_{0}$ with $G$-equivariant $K_{0}$-algebra homomorphism $K \rightarrow L$, respectively, to every $H$-Galois algebra over $L_{0}$. The equivalence of categories of the previous paragraph identifies these two non-abelian cohomology sets. For the compatibility, we use the category of $H$-Galois $L_{0}^{\prime} / L_{0}$ with extension of the $K_{0}$-algebra structure of $L_{0}$ to a $K$-algebra structure, with functor

$$
\left(L_{0}^{\prime} / L_{0}, K \stackrel{\beta}{\rightarrow} L_{0}\right) \mapsto\left(\left(\prod_{g \in G} L_{0}^{\prime}\right)^{H} / L_{0}, x \mapsto\left(\beta\left({ }^{g} x\right)\right)_{g \in G}\right)
$$

whose composite with the functor of the previous paragraph becomes naturally isomorphic to the forgetful functor after replacing $K$ by a suitable étale extension $\widehat{K}$. Functorial associations on this category, as above, are characterized by elements of $H^{1}\left(H, G L_{n}(\widehat{K})\right)$, such that the functors give 
rise to the relevant maps on non-abelian cohomology sets. We obtain the required compatibility.

Assumption 1 always holds when $k$ contains all roots of unity. In general, after performing the divisorialification procedure (see Section 4) the following stronger condition will hold.

Assumption 2. For all $H$ and all $k\left(Y_{i}\right) / k\left(Z_{i}\right)$ the field $k\left(Z_{i}\right)$ contains primitive $e$ th roots of unity, where $e$ is the exponent of $H$, and the composition of the map from Assumption 1 with the restriction

$$
\operatorname{Pic}^{G}(X) \rightarrow \operatorname{Pic}^{G}\left(\operatorname{Spec}\left(k\left(Y_{i}\right)\right)\right) \cong H^{1}\left(G, k\left(Y_{i}\right)^{\times}\right)
$$

is a surjective homomorphism

$$
\operatorname{Pic}^{G}(X) \rightarrow H^{1}\left(H, k\left(Z_{i}\right)^{\times}\right) .
$$

Proposition A.2. Let $k$ be a field of characteristic zero and $X$ a smooth projective variety over $k$ with a faithful action of a finite abelian group $G$. Then there exist smooth projective varieties with $G$-action and $G$ equivariant morphisms

$$
X^{\prime}=X_{n} \rightarrow \cdots \rightarrow X_{1} \rightarrow X_{0}=X
$$

each the blow-up of a smooth $G$-invariant subscheme, such that $X^{\prime} \oslash G$ satisfies Assumption 2.

Proof. The divisorialification procedure, applied to $[X / G]$, translates into a sequence of blow-ups that meets the stated conditions.

Proposition A.3. Let $k$ be a field of characteristic zero, $X$ and $X^{\prime}$ smooth projective varieties with faithful actions of a finite abelian group $G$ satisfying Assumption 2, and

$$
\varphi: X^{\prime} \rightarrow X
$$

a G-equivariant birational map restricting to an isomorphism over open $U \subset X$. Then there exists a weak factorization of $\varphi$, where each map is, or is inverse to, the blow-up of a smooth $G$-invariant subscheme disjoint from $U$ and the intermediate projective varieties with $G$-action satisfy Assumption 2.

Proof. There is no loss of generality in supposing $\varphi$ to be a morphism. By Assumption 2 there is a finite collection of $G$-linearized line bundles on $X$ whose classes in $\mathrm{Pic}^{G}(X)$ map to a generating system of $H^{1}\left(H, k\left(Z_{i}\right)^{\times}\right)$ for every $H$ and $k\left(Y_{i}\right) / k\left(Z_{i}\right)$. We represent these by a $\mathbb{G}_{m}^{r}$-torsor $V \rightarrow$ $X$ (where $r$ denotes the number of line bundles), with $G$-action on $V$ determined by the linearizations. By [5, Rmk. 7.14], Assumption 2 for $X$ implies that the $G$-action on $V$ is free. Let $V^{\prime}=X^{\prime} \times_{X} V$. Functorial 
weak factorization [3] provides compatible weak factorizations of $X^{\prime} \rightarrow X$ ( $G$-invariant, maintaining isomorphisms over $U$ ) and $V^{\prime} \rightarrow V$, with $\mathbb{G}_{m^{-}}^{r}$ torsor structure preserved throughout the weak factorization. Since, for any subgroup $H \subset G$, the property of having a point with stabilizer exactly $H$ is preserved under blow-up of a smooth $G$-invariant subscheme, we see that the freeness of the $G$-action is maintained throughout the weak factorization. By [5, Rmk. 7.14], again, we deduce that Assumption 2 is maintained throughout the weak factorization.

As stated in the Introduction, $\operatorname{Burn}_{n}(G)$ is a quotient of the free abelian group on triples consisting of a subgroup $H$ of $G$, a $G / H$-Galois algebra extension $K$ of a field $K_{0}$ of some transcendence degree $d \leq n$ over $k$ satisfying Assumption 1, and a faithful $(n-d)$-dimensional linear representation of $H$ over $K_{0}$ with trivial space of invariants. Any such representation splits as a sum of one-dimensional representations, so we may write the representation as a sequence of $(n-d)$ nonzero elements that generate the Cartier dual $\widehat{H}$. Triples related by an equivariant isomorphism of algebras over $k$ are regarded as equivalent, as are those which differ by a permutation of the elements of $\widehat{H}$. Then we identify

$$
\left[G / H \subset K,\left(a_{1}, \ldots, a_{n-d}\right)\right]
$$

with, for any $2 \leq j \leq n-d$, the sum over pairs $\left(I, C_{I}\right)$ with $\emptyset \neq I \subset$ $\{1, \ldots, j\}$ and $C_{I}$ a coset (of some subgroup) in $A$, satisfying

$$
I=\left\{1 \leq i \leq j \mid a_{i} \in C_{I}\right\}, \quad C_{I}=a_{i_{0}}+\left\langle a_{i}-a_{i_{0}}\right\rangle_{i \in I}\left(i_{0} \in I\right),
$$

of the generator indexed by the triple that we get as follows:

- Set $H_{I}=\bigcap_{\chi \in A_{I}} \operatorname{ker}(\chi)$, where $A_{I}=\left\langle a_{i}-a_{i_{0}}\right\rangle_{i \in I}$. So,

$$
\widehat{H_{I}}=A / A_{I} \text {. }
$$

- Let a representation of $H_{I}$ be determined by $a_{i_{0}}$ together with $a_{i}-a_{i_{0}}$ for all $i \leq j$ with $i \notin I$ and $a_{j+1}, \ldots, a_{n-d}$; this gives a sequence of elements of $A / A_{I}$ that is independent of $i_{0} \in I$.

- Writing $I=\left\{i_{0}, i_{1}, \ldots, i_{|I|-1}\right\}$ and letting

$$
b^{(i)}=\left(b_{g}^{(i)}\right)_{g \in G}
$$

denote the 1-cocycle with values in $K^{\times}$corresponding by Assumption 1 to $a_{i} \in \widehat{H}=H^{1}\left(H, K_{0}^{\times}\right)$, we let $G / H_{I}$ act on the field $K\left(t_{1}, \ldots, t_{|I|-1}\right)$ by the given action on $K$ and by $b^{\left(i_{c}\right)} / b^{\left(i_{0}\right)}$ on $t_{c}$, for $c=1, \ldots,|I|-1$.

It may happen, for some $\left(I, C_{I}\right)$, that 0 appears in the sequence of elements of $A / A_{I}$. Those $\left(I, C_{I}\right)$ are simply omitted from the sum, leaving a sum of generators associated with valid triples. 


\section{REFERENCES}

[1] D. Abramovich, T. Graber, and A. Vistoli. Gromov-Witten theory of DeligneMumford stacks. Amer. J. Math., 130(5):1337-1398, 2008.

[2] D. Abramovich, K. Karu, K. Matsuki, and J. Włodarczyk. Torification and factorization of birational maps. J. Amer. Math. Soc., 15(3):531-572, 2002.

[3] D. Abramovich and M. Temkin. Functorial factorization of birational maps for qe schemes in characteristic 0. Algebra Number Theory, 13(2):379-424, 2019.

[4] K. Behrend and B. Noohi. Uniformization of Deligne-Mumford curves. J. Reine Angew. Math., 599:111-153, 2006.

[5] D. Bergh. Functorial destackification of tame stacks with abelian stabilisers. Compos. Math., 153(6):1257-1315, 2017.

[6] D. Bergh and D. Rydh. Functorial destackification and weak factorization of orbifolds, 2019. arXiv:1905.00872.

[7] E. Bierstone and P. D. Milman. Canonical desingularization in characteristic zero by blowing up the maximum strata of a local invariant. Invent. Math., 128(2):207-302, 1997.

[8] F. Bittner. The universal Euler characteristic for varieties of characteristic zero. Compos. Math., 140(4):1011-1032, 2004.

[9] K. S. Brown. Cohomology of groups, volume 87 of Graduate Texts in Mathematics. Springer-Verlag, New York-Berlin, 1982.

[10] C. Cadman. Using stacks to impose tangency conditions on curves. Amer. J. Math., 129(2):405-427, 2007.

[11] C. U. Jensen, A. Ledet, and N. Yui. Generic polynomials: Constructive aspects of the inverse Galois problem. Cambridge University Press, Cambridge, 2002.

[12] S. Keel and S. Mori. Quotients by groupoids. Ann. of Math. (2), 145(1):193-213, 1997.

[13] S. L. Kleiman and A. B. Altman. Bertini theorems for hypersurface sections containing a subscheme. Comm. Algebra, 7(8):775-790, 1979.

[14] M. Kontsevich, V. Pestun, and Yu. Tschinkel. Equivariant birational geometry and modular symbols, 2019. arXiv:1902.09894.

[15] M. Kontsevich and Yu. Tschinkel. Specialization of birational types. Invent. Math., 217(2):415-432, 2019.

[16] A. Kresch. On the geometry of Deligne-Mumford stacks. In Algebraic geometrySeattle 2005. Part 1, volume 80 of Proc. Sympos. Pure Math., pages 259-271. Amer. Math. Soc., Providence, RI, 2009.

[17] A. Kresch. Destackification with restricted root operations. Eur. J. Math., 4(4):1421-1432, 2018.

[18] Yu. I. Manin. Parabolic points and zeta functions of modular curves. Izv. Akad. Nauk SSSR Ser. Mat., 36:19-66, 1972.

[19] I. Moerdijk. Orbifolds as groupoids: an introduction. In Orbifolds in mathematics and physics (Madison, WI, 2001), volume 310 of Contemp. Math., pages 205-222. Amer. Math. Soc., Providence, RI, 2002.

[20] J. Oesinghaus. Conic bundles and iterated root stacks. Eur. J. Math., 5(2):518$527,2019$.

[21] V. P. Platonov and A. S. Rapinchuk. Algebraicheskie gruppy i teoriya chisel. Nauka, Moscow, 1991.

[22] O. E. Villamayor U. Patching local uniformizations. Ann. Sci. École Norm. Sup. (4), 25(6):629-677, 1992. 
Institut FÜr MAThematik, Universität ZÜrich, Winterthurerstrasse 190, CH-8057 Zürich, SwitzerLAND

Email address: andrew.kresch@math.uzh.ch

Courant Institute, 251 Mercer Street, New York, NY 10012, USA

Email address: tschinkel@cims.nyu.edu

Simons Foundation, 160 Fifth Avenue, New York, NY 10010, USA 